\title{
The Therapeutic Effects of Curcumin in Early Septic Acute Kidney Injury: An Experimental Study
}

\author{
Shuo Wang iD $^{1,2}$ \\ Ping Zhao ${ }^{1,2}$ \\ Ying Zhang ${ }^{2}$ \\ Lianhua Zhu ${ }^{2}$ \\ Jianing Zhu ${ }^{1,2}$ \\ Yukun Luo ${ }^{2}$ \\ Qiuyang $\mathrm{Li}^{2}$
}

'Medical School of Chinese PLA, Chinese PLA General Hospital, Beijing, People's Republic of China; ${ }^{2}$ Department of Ultrasound, The First Medical Center of Chinese PLA General Hospital, Beijing, People's Republic of China
Correspondence: Yukun Luo; Qiuyang Li Department of Ultrasound, The First Medical Center of Chinese PLA General Hospital, Fuxing Road 28\#, Beijing, 100853, People's Republic of China Tel +86-10-5549-9056

Fax +86-10-6693-9533

Email lyk301@I63.com;

liqiuyang0925@163.com
Purpose: Sepsis is the leading condition associated with acute kidney injury (AKI) in the hospital and intensive care unit (ICU), sepsis-induced AKI (S-AKI) is strongly associated with poor clinical outcomes. Curcumin possesses an ability to ameliorate renal injury from ischemia-reperfusion, but it is still unknown whether they have the ability to reduce S-AKI. The aim of this study was to investigate the protective effects of curcumin on S-AKI and to assess its therapeutic potential on renal function, inflammatory response, and microcirculatory perfusion.

Methods: Male Sprague-Dawley (SD) rats underwent cecal ligation and puncture (CLP) to induce S-AKI and immediately received vehicle (CLP group) or curcumin (CLP+Cur group) after surgery. At 12 and $24 \mathrm{~h}$ after surgery, serum indexes, inflammatory factors, cardiac output (CO), renal blood flow and microcirculatory flow were measured.

Results: Serum levels of creatinine (Scr), cystatin C (CysC), IL-6 and TNF- $\alpha$ were significantly lower in the CLP+Cur group than those in the CLP group $(\mathrm{P}<0.05)$. Treatment with curcumin improved renal microcirculation at $24 \mathrm{~h}$ by measurement of contrast enhanced ultrasound (CEUS) quantitative parameters [peak intensity (PI); half of descending time (DT/2); area under curve (AUC); $\mathrm{P}<0.05]$. In histopathological analysis, treatment with curcumin reduced damage caused by CLP.

Conclusion: Curcumin can alleviate S-AKI in rats by improving renal microcirculatory perfusion and reducing inflammatory response. Curcumin may be a potential novel therapeutic agent for the prevention or reduction of S-AKI.

Keywords: curcumin, acute kidney injury, sepsis, microcirculation, inflammation, contrast enhanced ultrasound

\section{Introduction}

Sepsis is a systemic inflammatory response syndrome caused by infection, ${ }^{1}$ and it is the leading condition associated with acute kidney infection (AKI) in the hospital and intensive care unit (ICU). ${ }^{2}$ The annual global incidence of sepsis-induced AKI (S-AKI) is approximately 6 million cases or nearly 1 per 1000 population, ${ }^{3}$ and for ICU patients, sepsis is found in $40 \%$ to $50 \%$ of patients with AKI. ${ }^{4,5} \mathrm{~S}-\mathrm{AKI}$ is strongly associated with poor clinical outcomes. Early goal-directed therapy, such as fluid resuscitation and supportive treatment, can improve survival, ${ }^{6}$ however, an analysis of the Protocolized Care for Early Septic Shock (ProCESS) trial focused on renal outcomes found that the use of early goal-directed therapy or usual care did not influence new AKI development, severity of AKI, or renal recovery. ${ }^{7}$ It is becoming increasingly clear that restoring the macrocirculation is not always sufficient to adequately restore the microcirculation and preserve organ function. ${ }^{8}$ At the same time, inflammation associated with 
$\mathrm{S}-\mathrm{AKI}$ is coupled to long-term outcomes, as elevated plasma concentrations of inflammatory factors have been associated with nonrecovery of renal function and death in critically ill patients receiving renal replacement therapy (RRT). ${ }^{9}$ Thus, there is a pressing need for the development of novel therapeutic approaches that can restore perfusion of the renal microcirculation and exert anti-inflammatory effects. ${ }^{10,11}$

Curcumin (Cur, $\mathrm{C}_{21} \mathrm{H}_{20} \mathrm{O}_{6}$, Figure 1), the major active component of Curcuma longa, was first isolated in 1870 and widely used as spice, flavor, and colorant in daily life. ${ }^{12}$ Several studies have evidenced the antioxidant, antiinflammatory, anticarcinogenic, cardioprotective and nephroprotective effects of curcumin. ${ }^{13}$ The protective effects of curcumin on AKI are generally associated with its bifunctional antioxidant activity and anti-inflammatory activity. $^{14-16}$ Curcumin could ameliorate kidney disease with either acute or chronic nephritis, and reduce activation of the NF- $\kappa \mathrm{B}, \mathrm{MAPK}, \mathrm{AKT}$ and $\mathrm{pBAD}$ pathways either systemically, or within the inflamed kidneys. ${ }^{17}$ In addition, previous research found that curcumin could improve renal function during ischemia-reperfusion induced acute kidney injury, which protected the tubular epithelium from injury by modulating inflammatory processes, oxidative stress, and apoptosis. ${ }^{14}$ However, few studies have investigated the effects of curcumin on AKI induced by sepsis; whether or not curcumin can improve the renal microcirculation and exert anti-inflammatory effects is unclear. To investigate the therapeutic potential of curcumin, we established a septic rat model with AKI by cecal ligation and puncture (CLP), and then observed the changes in renal tissues and renal functions with or without curcumin intervention, and studied its effects on renal macro- and microcirculation and inflammatory reactions in septic rats.

\section{Materials and Methods}

\section{Animals}

Male Sprague-Dawley rats weighing $400 \mathrm{~g}$ to $500 \mathrm{~g}$ were housed in an animal facility with $12 \mathrm{~h}$ light-dark cycle at $22-24^{\circ} \mathrm{C}$. Rats were acclimated for $3-5$ days before conducting experiments with free access to food and water. All rats were purchased from the Laboratory Animal Center of the First Medical Center of Chinese PLA General Hospital (Experimental Animal License: SCXK (Beijing) 2019-0010). This study was approved by the Institutional Animal Care and Use Committee of the Chinese PLA General Hospital (2020-X16-76), and it was carried out in accordance with the Code of Practice for the Housing and Care of Animals Used in Scientific Procedures published by Her Majesty's Stationery Office.

\section{CLP Model of Sepsis}

A rat model of sepsis was established through cecal ligation and puncture (CLP) as described previously. ${ }^{18}$ Rats were anesthetized with $2 \%$ pentobarbital, and a 1 to $2 \mathrm{~cm}$ midline incision was made along the linea alba of the abdominal muscle to isolate and exteriorize the cecum. Fifty percent of the cecum was ligated with a 4 0 silk suture, and the cecum was punctured twice with a 16-gauge needle about $1 \mathrm{~cm}$ from the distal end. An approximately $1 \mathrm{~mm}$ column of fecal material was expressed. The cecum was then replaced to the peritoneal cavity and the abdomen was closed. In the sham group, rats underwent the same procedure but were neither ligated nor punctured. In the following surgery, all rats received $0.4 \mathrm{~mL} / \mathrm{kg}$ of prewarmed saline and were placed on a heating pad. All post-surgery rats were given free access to food and water.

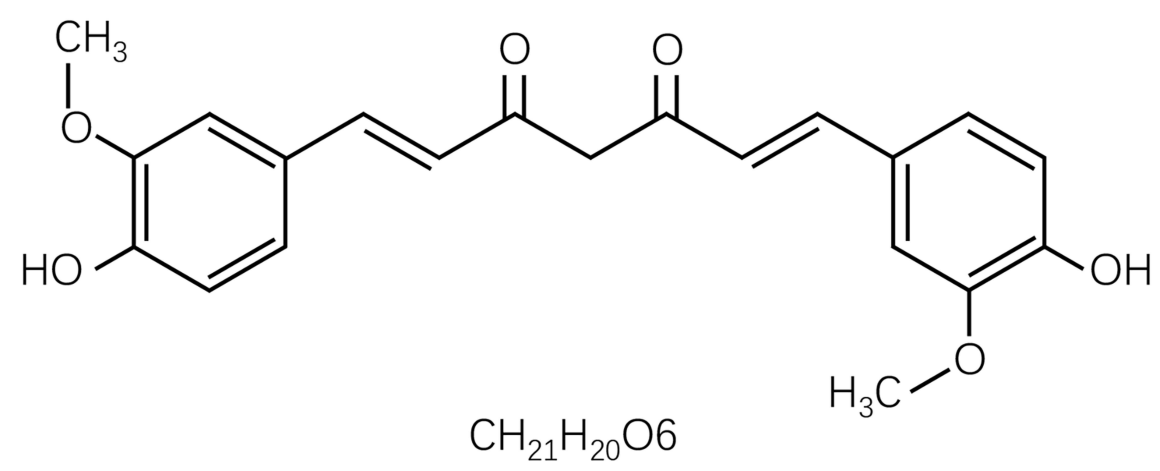

Figure I The chemical structure of curcumin. MW: 358.385 , BP: $591.4^{\circ} \mathrm{C}$, MP: $183^{\circ} \mathrm{C}$, density $\left(\mathrm{g} / \mathrm{m}^{3}, 25 / 4^{\circ} \mathrm{C}\right): 0.93$. 


\section{Animal Grouping}

Forty-eight rats were randomly and equally divided into three groups ( $\mathrm{n}=16$ for each group):

1. CLP+Cur group (Cur), which the rats received intraperitoneal injection with $100 \mathrm{mg} / \mathrm{kg}$ curcumin (Sigma, America) immediately after surgery.

2. CLP group (CLP), which the rats received intraperitoneal injection with vehicle (2.5\%DMSO) immediately after surgery.

3. Sham group (Sham), which the rats underwent identical surgical procedure but without any injection.

The curcumin solution applied in the experimental animals was freshly prepared. Immediately before the study, $500 \mathrm{mg}$ curcumin powder was dissolved with $2.5 \mathrm{~mL}$ of $100 \%$ dimethyl sulfoxide (DMSO, Sigma, America), and supplemented into $10 \mathrm{~mL}$ corn oil (Aladdin, China).

Twelve hours after the surgery, half of the animals in each group $(n=8)$ were sacrificed to collect serum from inferior vena cava and renal tissue under anesthesia.

Twenty-four hours after the surgery, the other half of the animals in each group $(n=8)$ were sacrificed with the same method.

\section{Assessment of Kidney Function}

Kidney injury was assessed by measuring the levels of serum creatinine (Scr), urea and serum cystatin $\mathrm{C}$ (CysC). Rats were euthanized at indicated time points under anesthetic. Blood samples were collected from the inferior vena cava; serum was separated by centrifugation at 3000 revolutions/min for $15 \mathrm{~min}$ and then stored at $-20^{\circ} \mathrm{C}$ until use. Scr, urea, and SysC were detected by automatic biochemistry analyzer (cobas 8000, Roche, Switzerland) according to manufacturer instructions. The results were recorded and analyzed.

\section{Enzyme-Linked Immunosorbent Assay}

The method of obtaining serum samples is the same as above. Serum levels of Interleukin-6 (IL-6), tumor necrosis factor- $\alpha$ (TNF- $\alpha$ ) and Interleukin-10 (IL-10) were measured using sandwich enzyme-linked immunosorbent assay according to manufacturer instructions (4Abio, China). Read plate at $450 \mathrm{~nm}$, then draw the standard line and analyze the data. Absorbance values at $450 \mathrm{~nm}$ were read and recorded by ELISA reader (Multiskan GO, Thermo, USA).

\section{Assessment of Hemodynamics}

Color Doppler ultrasound (CDFI) was fundamental for identifying vascularization, which can intuitively display the size and distribution of blood flow. Power Doppler (PD) is inherently more sensitive and can display flow in situations where CDFI is ineffective as it can display tissue perfusion in highly vascular organs such as the kidneys. ${ }^{19} \mathrm{PD}$ and CDFI were used to measure renal hemodynamics at $12 \mathrm{~h}$ and $24 \mathrm{~h}$ after CLP by a highresolution ultrasonic apparatus (M9t, Mindray, China) equipped with a linear array transducer (4-12 $\mathrm{MHz}$ ). Velocity scale, wall filter, and gain settings were held constant. Then the hemodynamics of kidney was assessed according to the size and distribution of blood flow in CDFI and PD images. M-mode echocardiography, an effective and efficient way to record the necessary multiple cardiac cycles, ${ }^{20}$ was performed to measure cardiac output (CO). $\mathrm{CO}$ was calculated as the product of stroke volume and heart rate.

\section{Assessment of Renal Microcirculation}

Contrast-Enhanced Ultrasound (CEUS), a method for detecting renal microcirculation, was performed by a highresolution ultrasonic apparatus (M9t, Mindray, China) equipped with a linear array transducer $(4-12 \mathrm{MHz})$ at a low mechanical index $(\mathrm{MI}=0.098)$. A maximum longitudinal scanning section that included the entire kidney was selected. Under contrast-enhanced mode, an intravenous infusion of $0.16 \mathrm{~mL} / \mathrm{kg}$ of SonoVue (Bracco, Italy) was administered to the caudal vein via bolus injection, followed by an immediate flush of $1 \mathrm{~mL}$ saline solution. SonoVue is a sulfur hexafluoride-filled microbubble encapsulated by a flexible phospholipid shell. The $2.5 \mu \mathrm{m}$ microbubbles cannot cross the endothelium; thus, the agent remains solely in the intravascular compartment. ${ }^{21}$ As soon as the contrast agent injection began, the CEUS process and the timer on the machine were started; realtime dynamic images were digitally stored at least $180 \mathrm{~s}$. Image depth, focus, gain, and frame rate were optimized and held constant for all further measurements.

CEUS images were quantitatively analyzed using the included software. Three similar-sized regions of interest (ROI) were drawn at the renal cortex and medulla, at the same approximate location and a similar depth while excluding interlobar and arcuate arteries. Signal time intensity curves (TIC) and blood perfusion parameters were automatically generated. The results of three ROIs 
at the renal cortex and medulla were averaged to minimize heterogeneity. CEUS parameters, arrival time (AT), time to peak (TTP), ascending slope (AS), peak intensity (PI), half of descending time (DT/2), descending slope (DS) and area under curve (AUC) were recorded and analyzed.

\section{Histopathology Assessments}

Kidneys were harvested and transversely incised in the center, fixed in $10 \%$ formalin, embedded in paraffin and then sectioned at $3 \mu \mathrm{m}$ thickness for staining with periodic acid-Schiff stain. Stained sections were observed by light microscopy (BX53F, OLYMPUS, Japan) at a magnification of 400X.

\section{Statistical Analyses}

Statistical analysis was performed using SPSS 26 (IBM Corporation, USA) and GraphPad Prism 8 software (GraphPad Software, USA). Results were evaluated using Shapiro-Wilk normality tests. For normally distributed data, the difference between experimental groups was shown using one-way ANOVA. Kruskal-Wallis tests were applied for data that did not show normal distribution. Tukey's test was used in multiple comparisons and $\mathrm{p}<0.05$ was considered as significant. Data showing normal are given as mean \pm standard error, those without normal distribution are given as $\mathrm{M}(\mathrm{IQR})$.

\section{Results}

\section{Effects of Curcumin on Renal Function}

The diagnosis of AKI is currently based on an increasing serum creatinine concentration. When creatinine $\geq 1.5$ times baseline, AKI occured. ${ }^{22}$ As shown in Figure 2 and Table 1 , at $12 \mathrm{~h}$ and $24 \mathrm{~h}$ after CLP, serum levels of Scr, urea and $\mathrm{CysC}$ were found significantly increased in the CLP group compared with the sham group $(\mathrm{P}<0.05)$, indicating the development of AKI. Apart from that, at $12 \mathrm{~h}$ after CLP, curcumin had no effect on the increase in
A

$12 \mathrm{~h} \mathrm{Scr}(\mu \mathrm{mol} / \mathrm{L})$

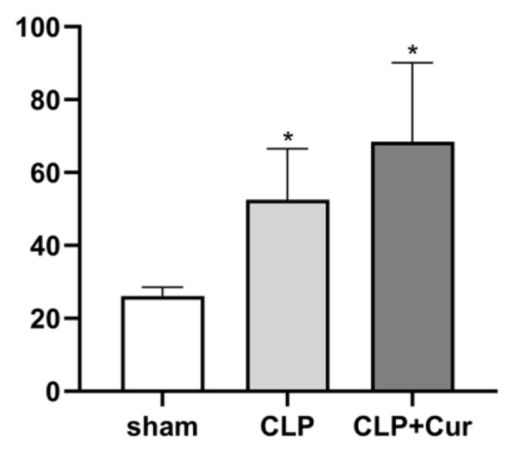

A

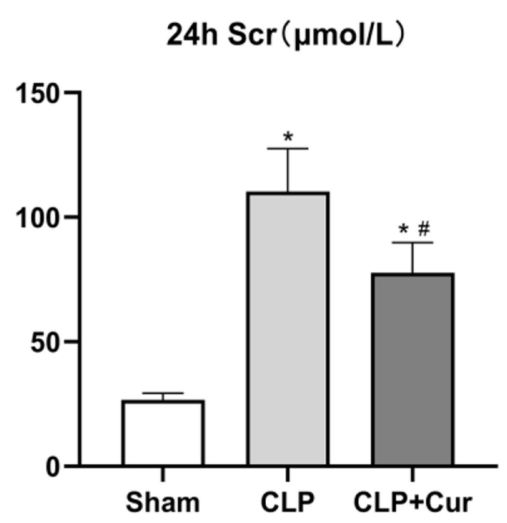

B

12h Urea(mmol/L)

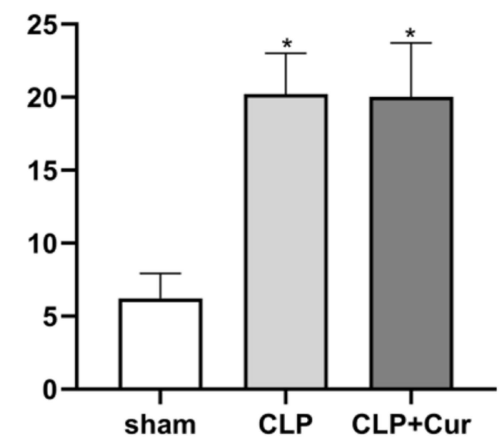

B

24h Urea(mmol/L)

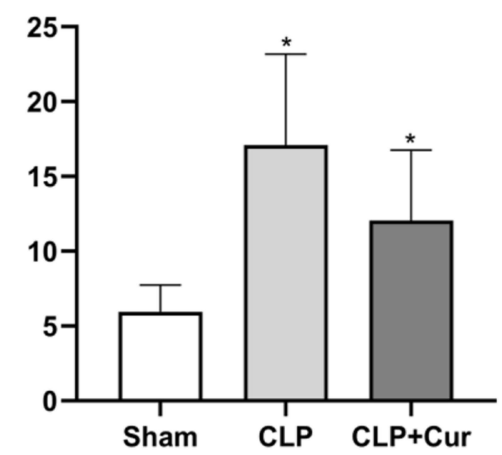

C

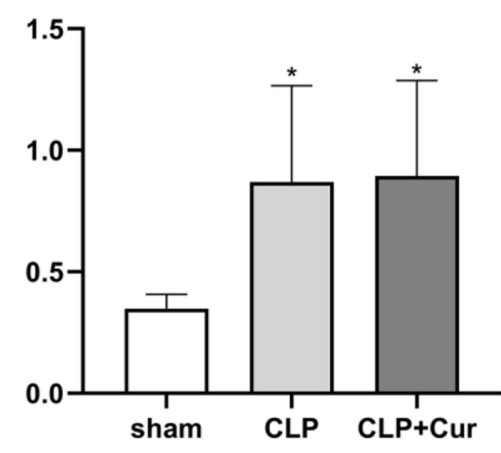

C

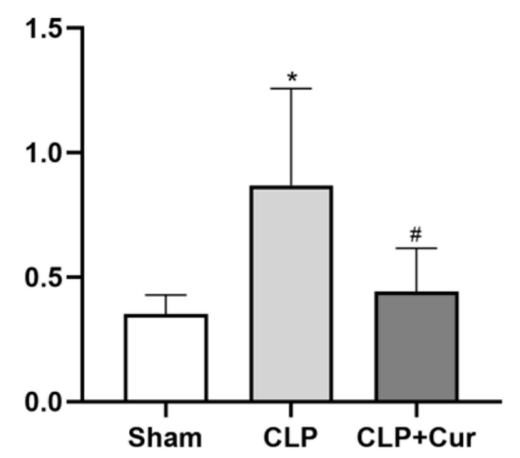

Figure 2 Effects of curcumin on serum Scr (a or A), Urea (b or B) and CysC (c or C) levels at I2h and 24h after CLP. At I2h and 24h after CLP, serum levels of Scr, Urea, and $\mathrm{Cys} C$ were increased compared with the sham group. At $24 \mathrm{~h}$ after $\mathrm{CLP}$, curcumin significantly reduced serum levels of Scr and Cys $\mathrm{C}$. $* \mathrm{P}<0.05$ compared with sham; $\# \mathrm{P}$ $<0.05$ compared with CLP ( $n=8$ per group). 
Table I Serum Indicators of Renal Function and Inflammation at 24h After Surgery

\begin{tabular}{|l|l|l|l|l|l|l|}
\hline & Scr $(\boldsymbol{\mu m o l} / \mathbf{L})$ & Urea $(\mathbf{m m o l} / \mathbf{L})$ & CysC $(\mathbf{m g} / \mathbf{L})$ & IL-6 $(\mathbf{p g} / \mathbf{L})$ & TNF- $\boldsymbol{\alpha}(\mathbf{p g} / \mathbf{L})$ & IL- $\mathbf{0}$ (pg/L) \\
\hline Sham & $26.68 \pm 0.95$ & $5.94 \pm 0.63$ & $0.35 \pm 0.03$ & $10.00 \pm 4.37$ & $8.94 \pm 1.70$ & $4.79 \pm 0.97$ \\
CLP & $110.31 \pm 6.08^{*}$ & $17.10 \pm 2.15^{*}$ & $0.87 \pm 0.14^{*}$ & $3170.55 \pm 785.13^{*}$ & $89.18 \pm 14.18^{*}$ & $464.74 \pm 169.37^{*}$ \\
CLP+Cur & $77.65 \pm 4.31^{* \#}$ & $12.06 \pm 1.66^{*}$ & $0.44 \pm 0.06^{\#}$ & $240.45 \pm 170.86^{* \#}$ & $33.22 \pm 7.5^{*}$ & $152.13 \pm 35.97^{*}$ \\
\hline
\end{tabular}

Notes: ${ }^{*} \mathrm{P}<0.05$ compared with sham; ${ }^{\#} \mathrm{P}<0.05$ compared with CLP ( $\mathrm{n}=8$ per group).

all indicators; while at $24 \mathrm{~h}$ after CLP, curcumin administration dramatically reduced Scr and CysC levels compared with CLP alone, there was a significant difference between the two groups $(\mathrm{P}<0.05)$.

\section{Effects of Curcumin on Inflammatory Cytokines}

After sepsis by CLP, there was an initial increase in cytokines within the first few hours. ${ }^{23}$ The concentration of IL-6, IL-10 and TNF- $\alpha$ are shown in Figure 3 and Table 1 in order to investigate the inflammation induced by sepsis. At $12 \mathrm{~h}$ and $24 \mathrm{~h}$ after CLP, serum IL-6, TNF$\alpha$, IL-10 were significantly increased compared with Sham group $(\mathrm{P}<0.05)$. At $24 \mathrm{~h}$ after CLP, there was a significant difference on IL-6 and TNF- $\alpha$, between CLP and CLP+Cur groups $(\mathrm{P}<0.05)$, which suggested that curcumin treatment had an effect on the decrease in serum inflammation cytokines.
A

$12 \mathrm{~h} \mathrm{Scr}(\mu \mathrm{mol} / \mathrm{L})$

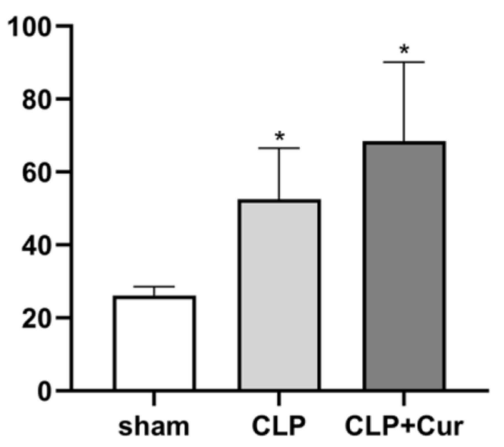

A

$24 \mathrm{~h} \mathrm{Scr}(\mu \mathrm{mol} / \mathrm{L})$

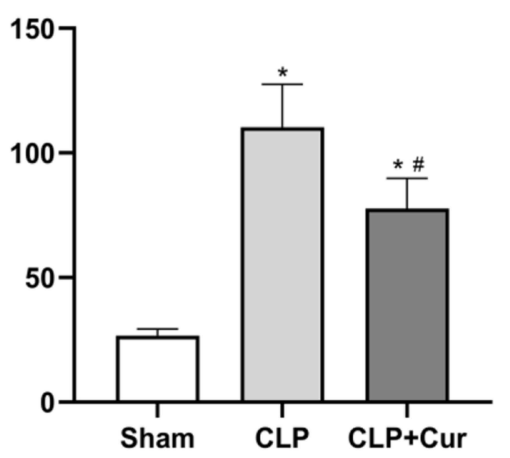

B

12h Urea(mmol/L)

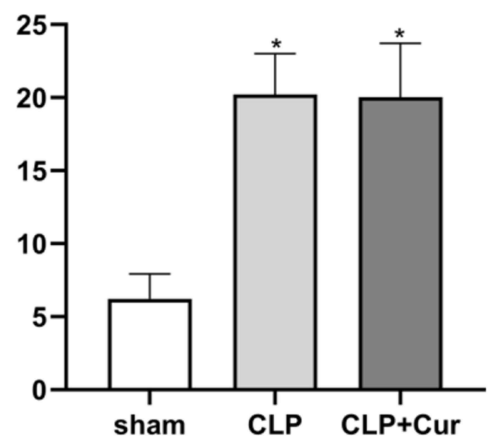

B

24h Urea(mmol/L)

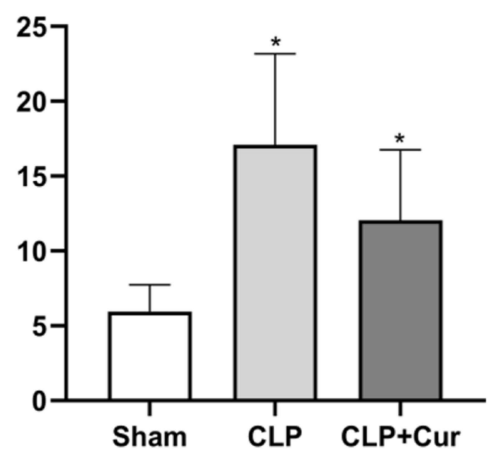

C

12h Cysc (mg/L)

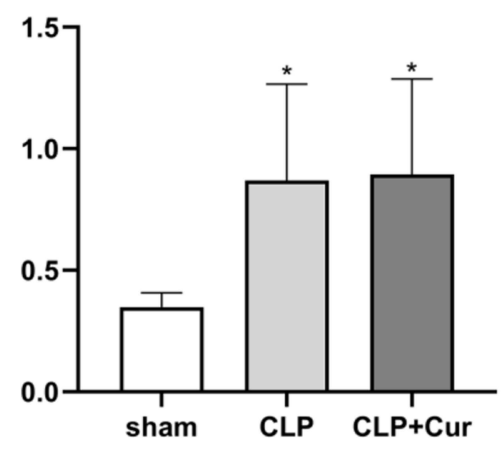

C

24h Cysc(mg/L)

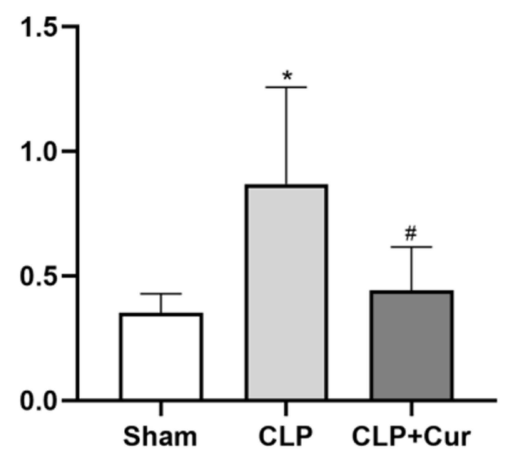

Figure 3 Effects of curcumin on serum IL-6 (a or A), TNF- $\alpha$ (b or B) and IL-10 (c or C) levels at I2h and 24h after CLP. At I2h and 24h after CLP, serum levels of IL-6, TNF- $\alpha$, and IL-10 were increased compared with the sham group. At $24 \mathrm{~h}$ after CLP, treatment with curcumin significantly reduced serum levels of IL-6 and TNF- $\alpha$. $* \mathrm{P}<0.05$ compared with sham; \#P $<0.05$ compared with CLP ( $=8$ per group). 


\section{Effects of Curcumin on Hemodynamics}

At $12 \mathrm{~h}$ and $24 \mathrm{~h}$ after CLP, CO was decreased compared with the sham group. These data indicate that curcumin treatment had no effect on the decrease of $\mathrm{CO}$ at either time point (Figures 4 and 5). As shown in Figure 6, the renal blood flow in CLP+Cur group is better than that in CLP group, the blood flow at $24 \mathrm{~h}$ after CLP was better than that $12 \mathrm{~h}$ after CLP. In the Sham group at $12 \mathrm{~h}$ and $24 \mathrm{~h}$, segmental artery, interlobar artery and interlobular artery could be clearly displayed. In CLP and CLP+Cur group at $12 \mathrm{~h}$, the intensity of blood flow signal was weakened and the interlobular artery was not clear, which indicates the construction of renal blood flow. At $24 \mathrm{~h}$ after surgery, the intensity of blood flow signal in CLP and CLP + Cur were increased compared with the two groups at $12 \mathrm{~h}$, and the signal of interlobular artery in CLP+Cur group at $24 \mathrm{~h}$ was clearer than CLP group. These data indicate that curcumin has no effect on cardiac output, but can improve renal blood flow.

\section{A}

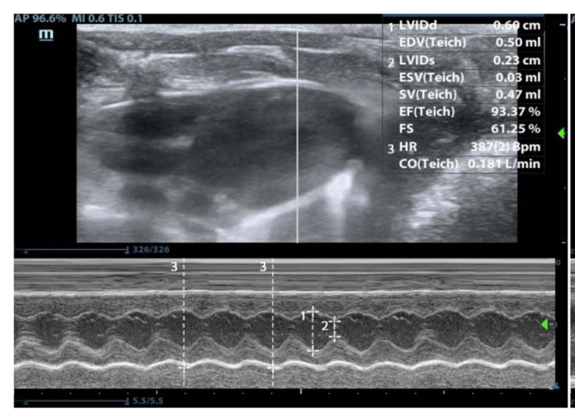

B
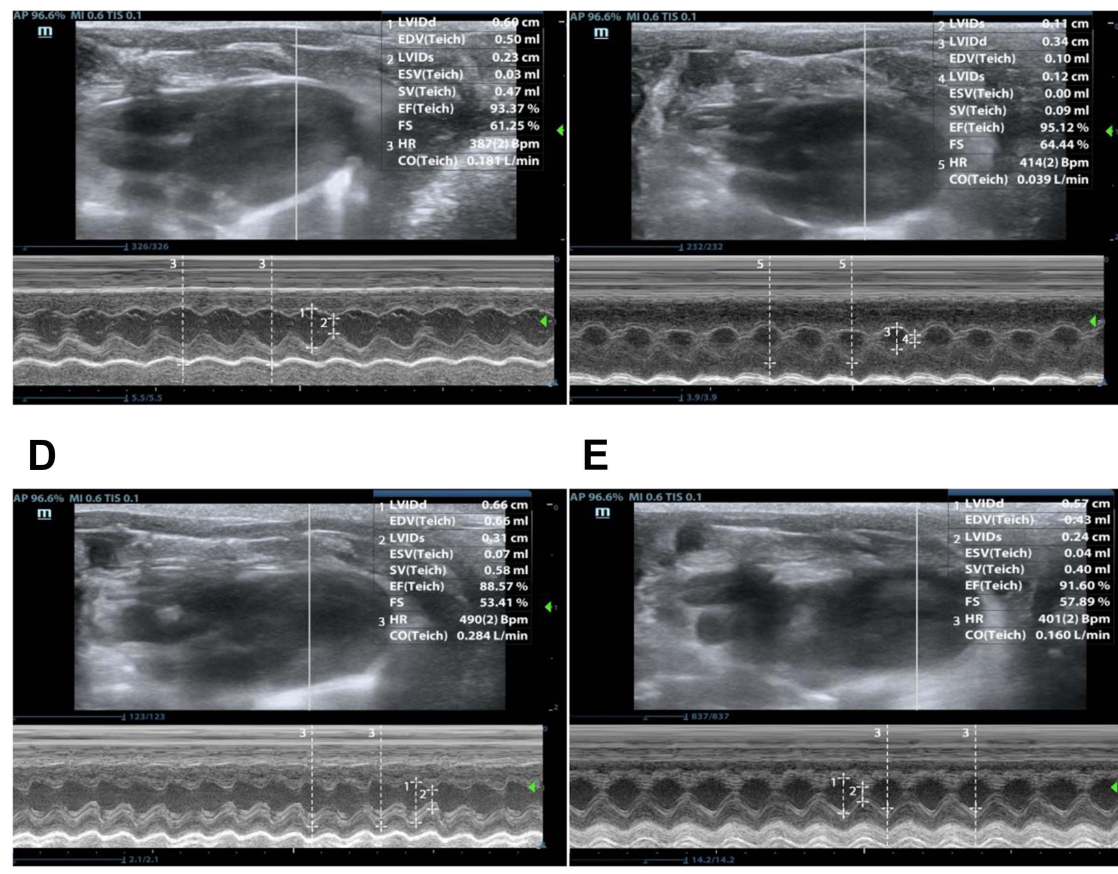

E

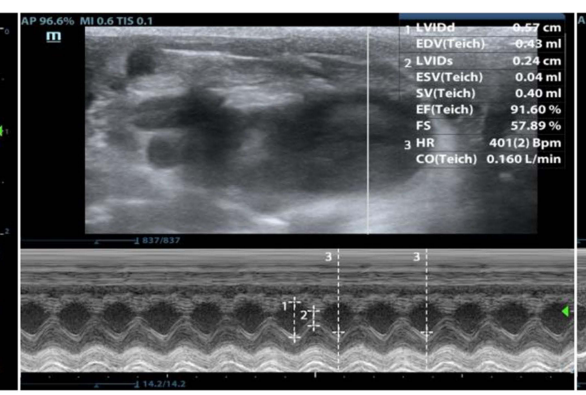

C

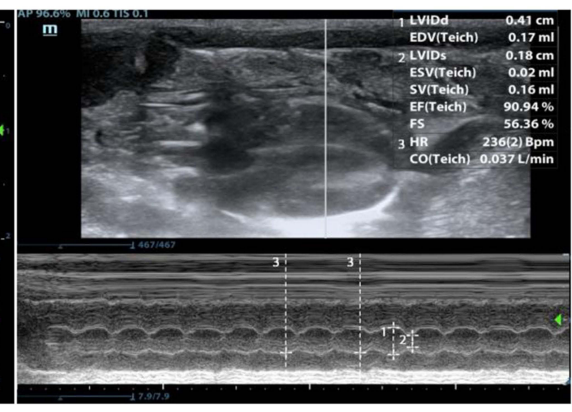

$\mathbf{F}$

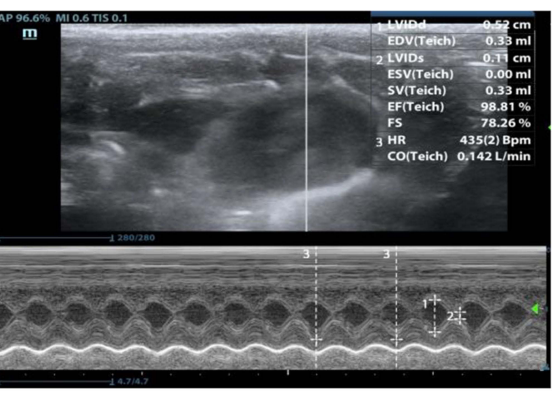

Figure 4 Heart M-mode ultrasound images. (A) Sham group at I2h. (B) CLP group at I2h. (C) CLP+Cur at I2h. (D) Sham group at 24h. (E) CLP group at 24h. (F) CLP + Cur at $24 \mathrm{~h}$. The stroke volume and heart rate were measured by M-mode ultrasound, and then the cardiac output was calculated.

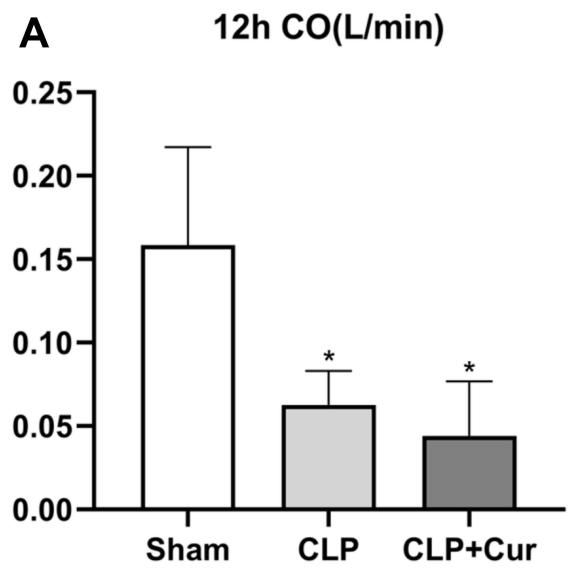

B

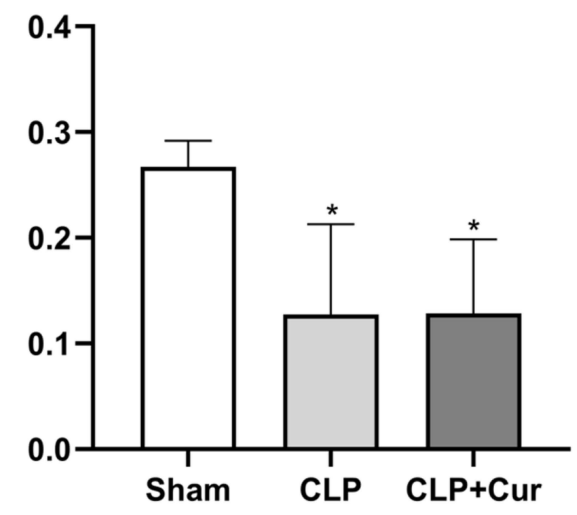

Figure 5 Effects of curcumin on cardiac output at I2h (A) and 24h (B) after CLP. At I2h and 24h after CLP, CO was decreased compared with the sham group. Curcumin had no effect on the decrease of $\mathrm{CO}$ at either time point. $* \mathrm{P}<0.05$ compared with sham; ${ }^{\#} \mathrm{P}<0.05$ compared with CLP ( $\mathrm{n}=8$ per group). 
A B

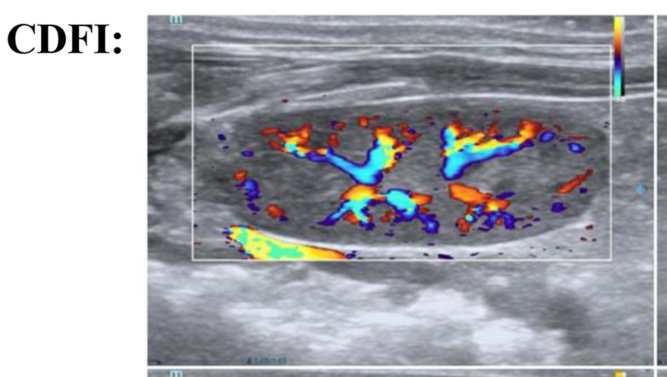

PD:

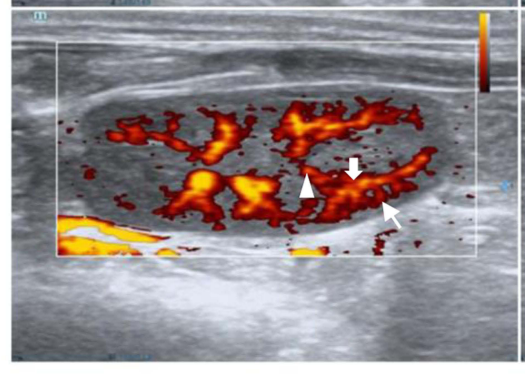

A

CDFI:

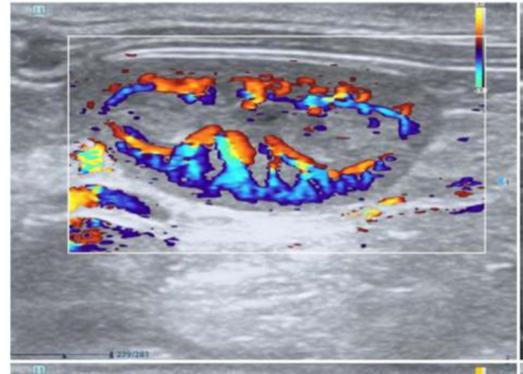

PD:

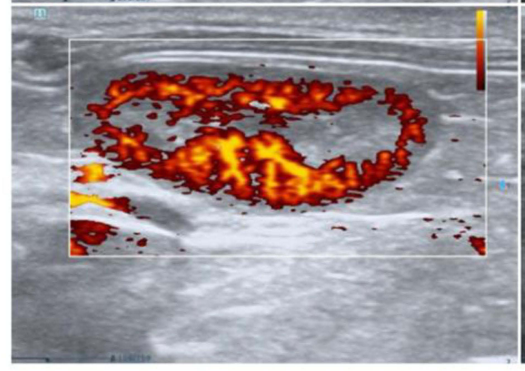

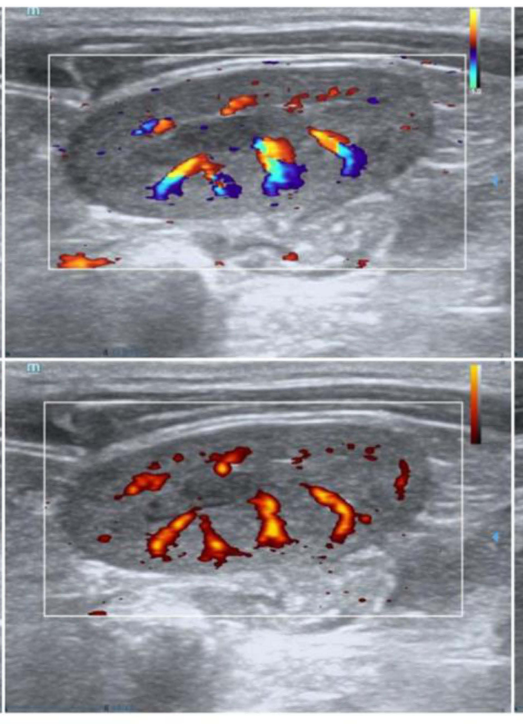

B
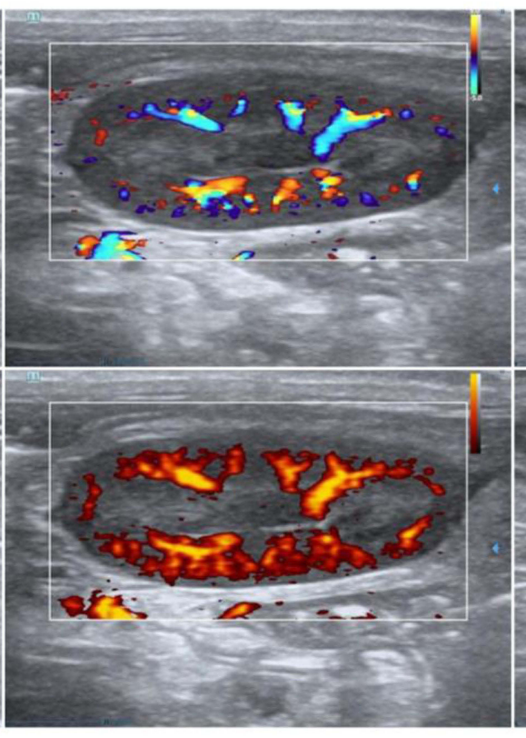

C

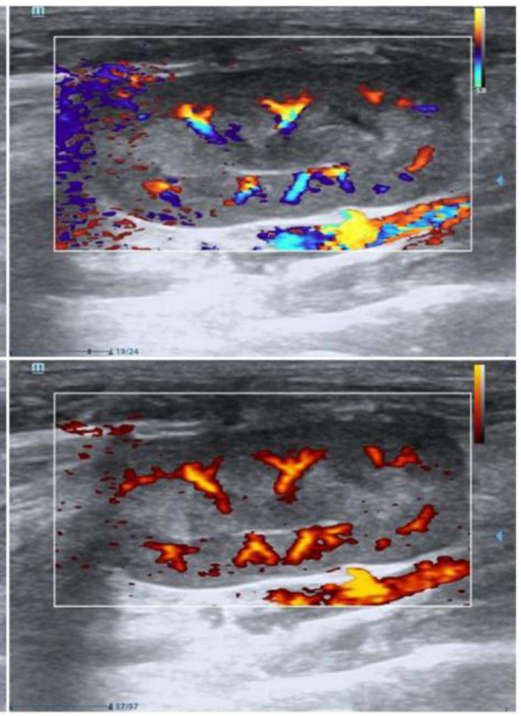

C

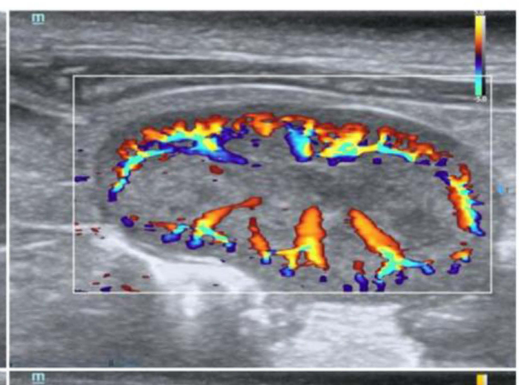

Figure $\mathbf{6}$ The comparison of Sham group (a and A), CLP group (b and $\mathbf{B}), \mathrm{CLP}+\mathrm{Cur}$ group $(\mathbf{C}$ and $\mathbf{c})$ using renal Power Doppler and CDFI. $\mathbf{\Delta}$ segmental artery, $\Downarrow$ interlobar artery, \interlobular artery. Overall, blood flow at $24 \mathrm{~h}$ after CLP $(\mathbf{A}-\mathbf{C})$ was better than $12 \mathrm{~h}$ after CLP (a-c). The Sham group was better than CLP+Cur group and CLP + Cur group was better than CLP group. Power Doppler is more sensitive in flow detection than CDFI ( $\mathrm{n}=8$ per group).

\section{Effects of Curcumin on Renal Microcirculation}

CEUS quantitative parameters reflect the perfusion sequence, time, intensity and distribution of renal microcirculation. 2-4 s after SonoVue bolus injection, renal blood vessels were enhanced, then the cortex and medulla developed; 1-2 min later, the medulla and cortex disappeared (Figure 7).
Renal microcirculation perfusion was significantly decreased compared with the sham group; curcumin treatment significantly increased perfusion of renal cortex at $24 \mathrm{~h}$ after CLP. At $12 \mathrm{~h}$, cortical quantitative parameters of PI in CLP group were significantly decreased compared with the sham group; curcumin treatment significantly enhanced the level of PI compared with CLP. DS in CLP group was significantly decreased compared with the sham 
A

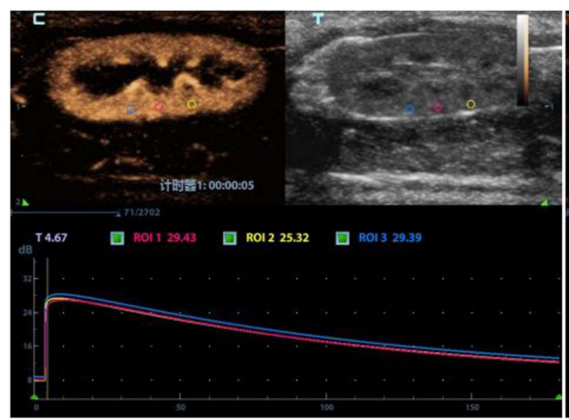

D

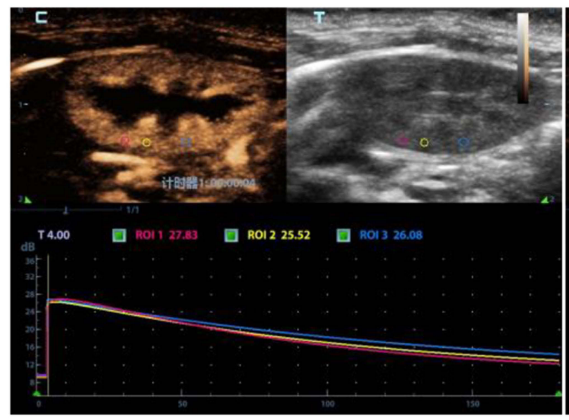

B

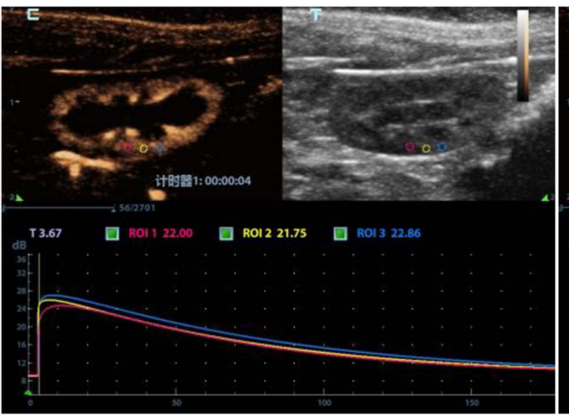

E

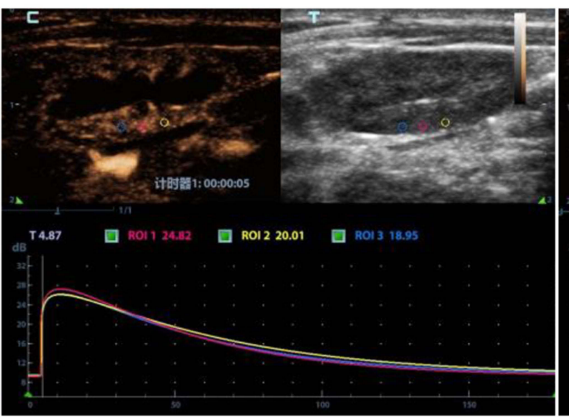

C

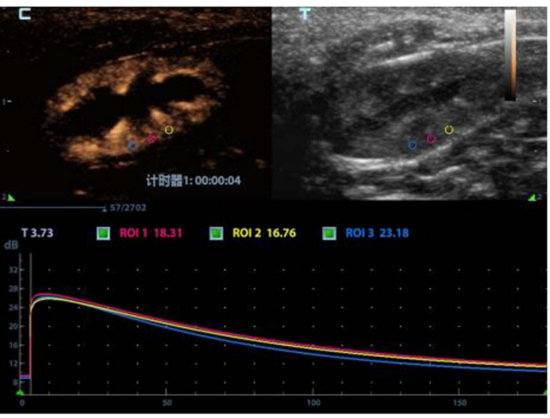

$\mathbf{F}$

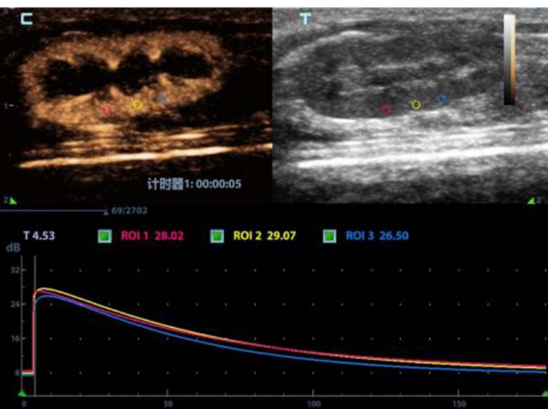

Figure 7 Contrast-Enhanced Ultrasound images. Perfusion of renal vessels, cortex and medulla and time intensity curve about the ROI. The perfusion in CLP group was weaker than that in CLP+Cur group from a macro view. (A) Sham group at I2h. (B) CLP group at I2h. (C) CLP+Cur at I2h. (D) Sham group at 24h. (E) CLP group at 24h. (F) CLP+Cur at 24h.

group, Curcumin treatment had no effect on the decrease of DS (Figure 8). At 24h, cortical levels of AUC, PI, DT/2 and DS in the CLP group were significantly decreased compared with the sham group, AS levels were significantly increased compared with the sham group. Curcumin treatment significantly increased levels of AUC, PI and
DT/2 compared with the CLP group and had no effect on the increase of DS or the decrease of AS. Renal medullary levels of DT/2, DS, and AUC in CLP group were significantly decreased compared with the sham group. Curcumin treatment had no effect on the decrease of DT/ 2, DS, or AUC (Figure 9 and Table 2).

\section{A}

cortex 12h PI(dB)

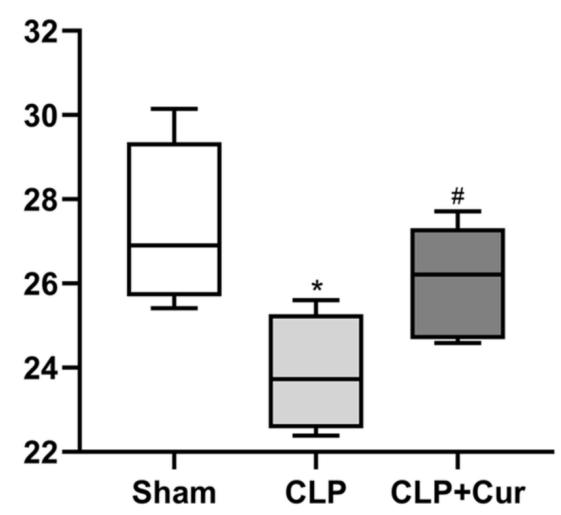

B

\section{cortex $12 \mathrm{~h} \mathrm{DS}(\mathrm{dB} / \mathrm{s})$}

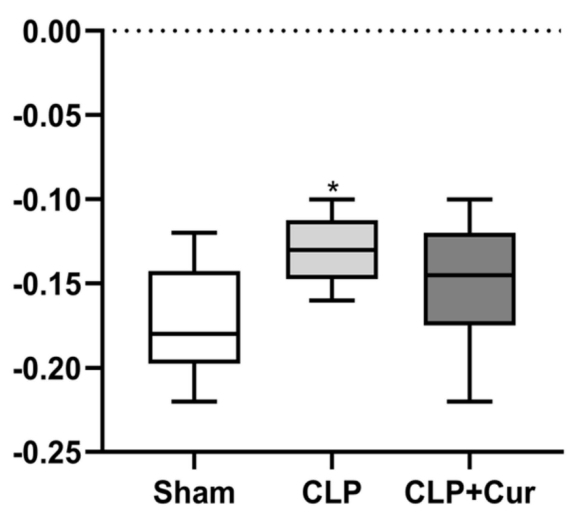

Figure 8 Changes in CEUS values and effects of curcumin at I2h. (A) PI in CLP group was significantly decreased compared with the sham group and curcumin treatment significantly enhanced the level of PI compared with CLP (B) DS in CLP group was significantly decreased compared with the sham group, *P < 0.05 compared with sham; ${ }^{\#} \mathrm{P}<0.05$ compared with CLP $(\mathrm{n}=8$ per group). 
A

cortex 24h AUC $(\mathrm{dB} \cdot \mathrm{s})$

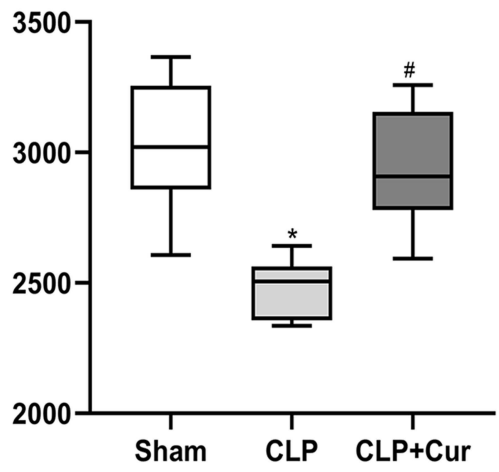

D

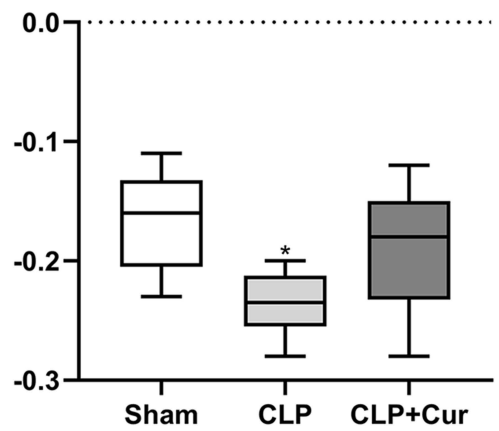

A

medulla 24h DT/2(s)

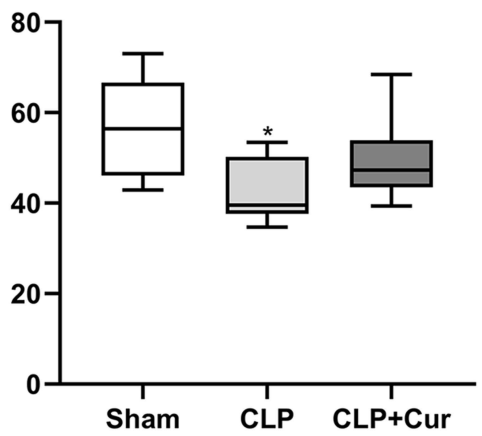

B

cortex 24h PI(dB)

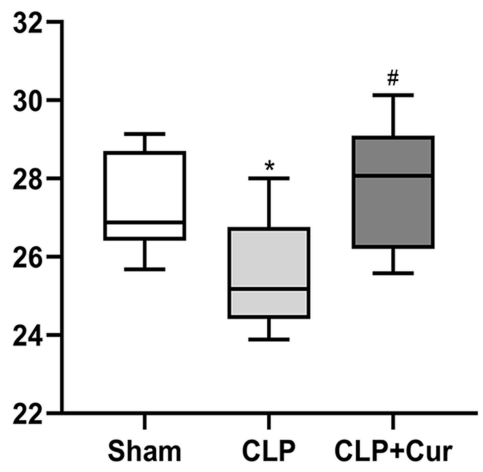

E

cortex 24h AS (dB/s)

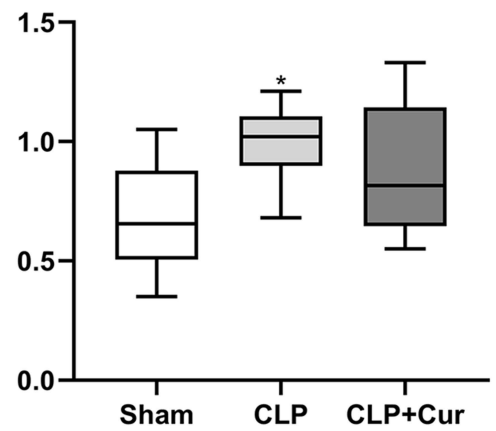

B

medulla 24h DS(dB/s)

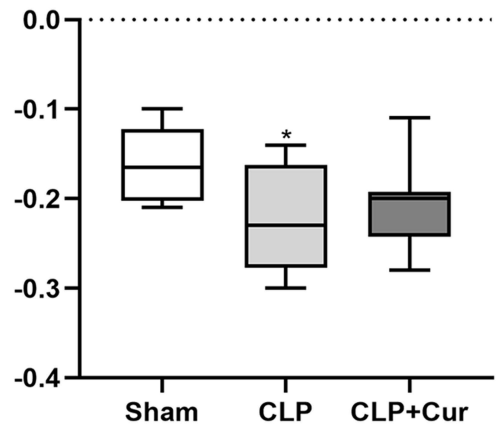

C

cortex 24h DT/2(s)

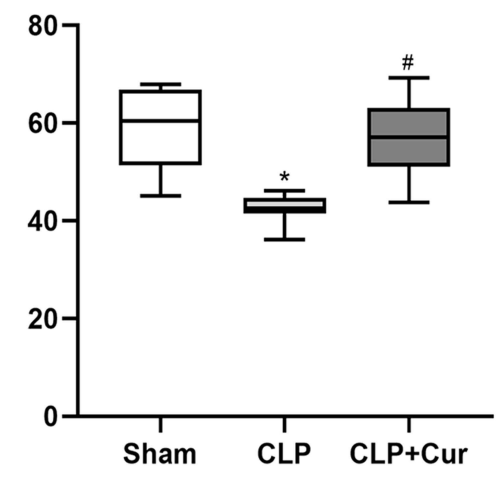

Figure 9 Changes in CEUS values and effects of curcumin at 24h. (a-e) CEUS values in renal cortex. Levels of AUC (a), PI (b), DT/2 (c) and DS (d) in the CLP group were significantly decreased compared with the sham group, AS (e) levels were significantly increased compared with the sham group. Curcumin treatment significantly increased levels of AUC, PI and DT/2 compared with the CLP group and had no effect on the increase of DS or the decrease of AS. (A-C) CEUS values in renal medulla. Levels of DT/ 2 (A), DS (B), AUC (C) in CLP group were significantly decreased compared with the sham group. Curcumin treatment had no effect on the decrease of DT/2, DS, AUC. *P $<0.05$ compared with sham; ${ }^{\#} P<0.05$ compared with CLP ( $n=8$ per group).

\section{Histopathological Results}

As seen in PAS-stained images, at 12 and 24h, kidney sections of the sham group revealed normal histological structure with malpighian bodies and tubular sections. Dilatation and unclear outline of renal tubules, shedding of brush margin, flattening and shedding of renal tubular epithelial cells were observed in 
Table 2 Quantitative Parameters of CEUS in Renal Cortex at 24h After Surgery

\begin{tabular}{|l|l|l|l|l|l|l|l|}
\hline & AT (s) & TTP (s) & PI (dB) & AS (dB/s) & DT/2 (s) & DS (dB/s) & AUC (dB s) \\
\hline Sham & $3.7 I \pm 0.93$ & $8.29 \pm 0.33$ & $27.36 \pm 0.45$ & $0.69 \pm 0.08$ & $58.72 \pm 2.99$ & $-0.17 \pm 0.15$ & $3028.31 \pm 87.37$ \\
CLP & $3.78 \pm 0.18$ & $8.21 \pm 0.45$ & $25.54 \pm 0.49 *$ & $0.99 \pm 0.59 *$ & $42.41 \pm 1.07^{*}$ & $-0.23 \pm 0.0 I^{*}$ & $2477.89 \pm 40.08^{*}$ \\
CLP+Cur & $3.73 \pm 0.07$ & $9.75 \pm 0.58$ & $27.81 \pm 0.56^{\#}$ & $0.89 \pm 0.10$ & $56.87 \pm 2.82^{\#}$ & $-0.19 \pm 0.02^{*}$ & $2945.99 \pm 80.23^{\#}$ \\
\hline
\end{tabular}

Notes: ${ }^{*} \mathrm{P}<0.05$ compared with sham; ${ }^{*} \mathrm{P}<0.05$ compared with CLP ( $\mathrm{n}=8$ per group).

kidney sections of the CLP group, parts of renal tubules were formed at 24h in CLP group. Images of CLP+Cur sections showed decreased damage compared to the CLP group at $24 \mathrm{~h}$ (Figure 10).

\section{Discussion}

At present, the pathogenesis of S-AKI is not fully understood. Therefore, treatment remains reactive and nonspecific, and no available preventive therapies exist. ${ }^{3}$ In terms of mechanism, inflammatory responses and microcirculatory alterations were two major theories. ${ }^{3,24,25}$ Circulating toxins act on the endothelium, triggering a reduction in microcirculatory flow and interstitial infiltration of inflammatory cells; ${ }^{26}$ inflammatory mediators are released, which triggers tubular cell stress and injury. ${ }^{27} \mathrm{~A}$ dysregulated inflammatory response

\section{A}

B

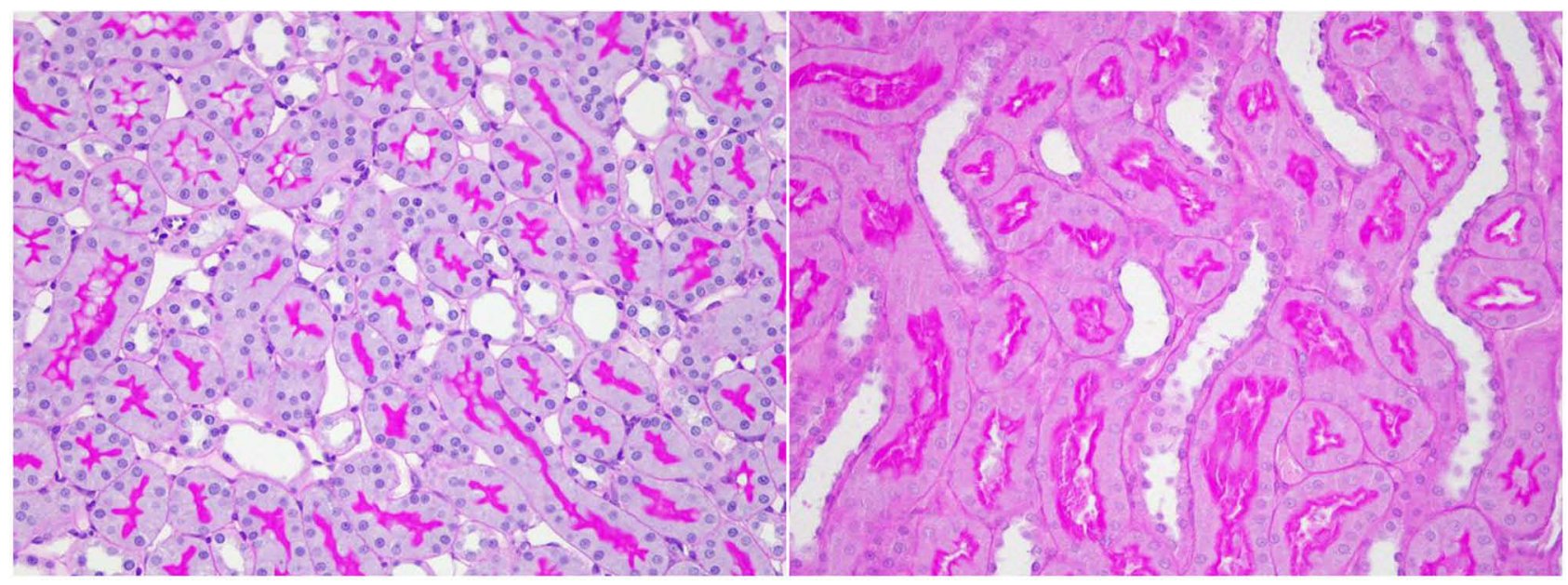

C

D

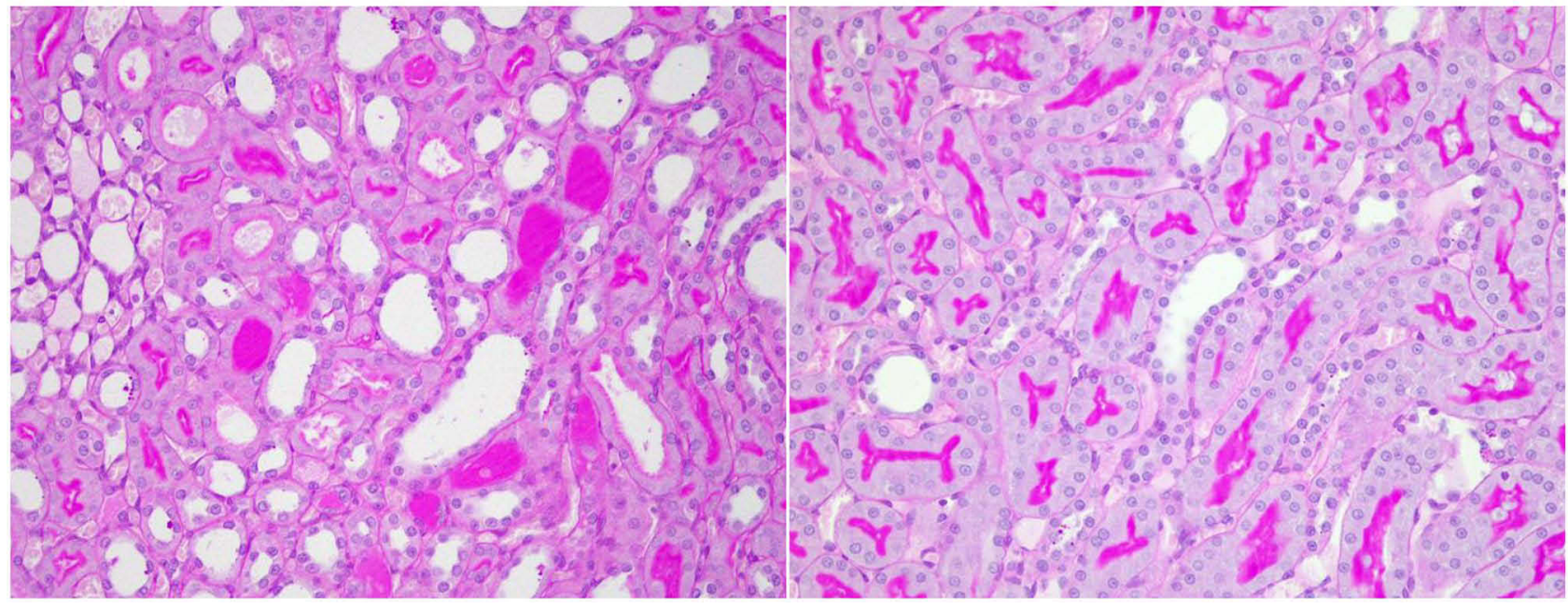

Figure 10 Light microscopy images of PAS-stained renal tissue $(\times 400)$. (A) Sham group; (B) CLP group, dilatation of renal tubules, shedding of brush margin, flattening and shedding of renal tubular epithelial cells; (C) CLP group, renal tubules were formed (D) CLP+Cur group, the degree of damage was lesser than that of CLP group. 
could be of great significance to organ dysfunction and poor outcome; ${ }^{3}$ microcirculatory alterations may play a key role in the development of organ injury. ${ }^{28}$ Previous studies showed that curcumin could protect renal function on AKI induced by ischemia/reperfusion $(\mathrm{I} / \mathrm{R}){ }^{14}$ we studied the therapeutic potential of curcumin using a rat sepsis model induced by CLP to investigate whether curcumin could regulate inflammation response and microcirculatory flow in S-AKI.

The diagnosis of AKI is currently based on an increase in serum creatinine concentration and/or a decrease in urine output. ${ }^{22}$ At 12 and $24 \mathrm{~h}$ after CLP, the level of Scr conforms to the diagnostic criteria of AKI. At $24 \mathrm{~h}$ after CLP, treatment with curcumin reduced serum levels of Scr and $\mathrm{CysC}$, suggest that curcumin can protect against renal dysregulation of S-AKI and has better effect than at $12 \mathrm{~h}$ after CLP. Though having a larger dataset such as NAGL and KIM-1 would offer further insight into kidney injury, the increase in Scr and urea was sufficient evidence that kidney was injured.

After CLP, proinflammatory cytokines TNF- $\alpha$ and IL-6 were released during the first few hours and competed with anti-inflammatory factors such as IL-10, which is closely related to the occurrence of renal injury. ${ }^{23}$ In our study, at $24 \mathrm{~h}$ after CLP, treatment with curcumin inhibited IL-6, TNF$\alpha$. Benes et $\mathrm{al}^{11}$ showed that in contrast to AKI-free animals, the development of septic AKI was preceded by early and remarkable inflammatory response (elevated TNF-a, IL-6), which is consistent with the findings in our CLP groups. In our study, curcumin did not reduce the serum level of IL-10. It has been proposed that endogenous IL-10 serves as a protective function in models of endotoxic shock; ${ }^{29}$ thus, curcumin does not destroy this protective effect.

Previous studies showed that in humans and large animal models, including sheep and pigs, $\mathrm{CO}$ was usually increased during the initial stage of sepsis. ${ }^{30-32}$ In our study, $\mathrm{CO}$ and renal flow observing by CDFI and Power Doppler in CLP and CLP+Cur group were decreased compared with the sham group. We considered there may be a difference in $\mathrm{CO}$ between large animals and rodents. We established a severe sepsis animal model by adjusting the number of perforations and the location of ligation, which leads to septic shock in experimental animals, resulting in a decrease in blood flow. In our study, curcumin treatment has no effect on the decrease of CO.

Contrast-enhanced ultrasound (CEUS) is a new technique which enables the observation of blood perfusion and can provide real-time, noninvasive and relative quantitative estimates of renal microvascular perfusion independent of kidney function. ${ }^{33}$ It has been tested in critically ill patients, including S-AKI. When S-AKI occurred, there were profound heterogeneous changes in microcirculatory flow. ${ }^{3}$ ROIs were selected to draw a time intensity curve (TIC) and perform quantitative analysis. Arrival time (AT), ascending slope (AS), peak intensity (PI), time to peak (TTP), DT/2, descending slope (DS), and area under the curve (AUC) are the key observational parameters used in renal perfusion studies. PI is intensity parameter, which represents the maximum intensity of the curve. AT, TTP, DT/2 are time parameters. AT is the time when contrast agents arrive; TTP represents the time to maximum enhancement; DT/ 2 is the time from injection until decay to the half peak of enhancement. AS, DS, and AUC are time-intensity parameters, AS is the wash-in slope of the contrast agents, and reflects the perfusion velocity of contrast agents. DS is the wash-out slope of the contrast agents, and reflects the clearance velocity of the contrast agents; AUC is the area under the TIC, which is proportional to the total volume of blood flow in the ROI. In our study, cortical quantitative parameters of PI, DS at $12 \mathrm{~h}$; cortical parameters of AS, PI, DT/2, DS, AUC and medullary parameters of DT/2, DS, AUC at $24 \mathrm{~h}$ are significantly different between the sham group and CLP group. The decrease in PI and AUC indicates a decrease in blood flow volume; when microcirculatory flow is decreased, the speed of perfusion and clearance is increased, and the time and PI are decreased. The quantitative parameters result of our study is consistent with it, which suggests that the renal microcirculatory flow decreases during $\mathrm{S}-\mathrm{AKI}$ in rats, and further, the condition at $24 \mathrm{~h}$ was more serious than at $12 \mathrm{~h}$. Previous study observed the decrease in cortical renal perfusion during $\mathrm{S}-\mathrm{AKI},{ }^{34}$ which was consistent with our study and demonstrated the rationality of our model. Multiple mechanisms may lead to microcirculatory alterations, such as endothelial injury and activation of the coagulation cascade. ${ }^{35}$ Treatment with curcumin significantly increased the cortical quantitative parameters of PI at 12h, and PI, DT/2, AUC at 24h, indicating that curcumin significantly increased the renal microcirculation perfusion in CLP rats. From the results of the histopathological sections, it was clear that after curcumin intervention, damage in renal tubular epithelial cells was improved.

Although the role of the curcumin is recognized in AKI, we have explored the protective effects on the 
circulation perfusion during treatment of S-AKI for the first time. In our study, we found that the curcumin has an excellent effect on anti-inflammation, which is consistent with previous studies. ${ }^{14,15}$ The mechanism of the antiinflammation may be that curcumin reduced inflammatory processes via the semaphorin-plexin pathway and suppressed NF- $\kappa \mathrm{B}$ mediating inflammation by activating JAK2/STAT3 signal pathway. ${ }^{14,15}$ When sepsis occurs, circulating toxins might act on the endothelium, which triggers reduced microcirculatory flow and interstitial infiltration of inflammatory cells. ${ }^{26}$ Curcumin is known to have an active biochemical impact on the endothelial protection, which may exert a protective effect on endothelium-based vascular regulation by regulating the intactness of microvascular endothelium, ${ }^{36}$ as a result, improving renal microcirculation. The evaluation of AKI needs to be based on the combination of serum renal function and hemodynamics. ${ }^{37}$ In our multiple evaluation of early S-AKI, curcumin exerted therapeutic effects in it.

\section{Conclusion}

In our study, we show that curcumin represents a new and promising effective treatment in S-AKI rat models. Treatment with curcumin ameliorates renal functions, improves both renal macro- and microcirculatory flow, reduces inflammatory response and prevents pathological changes in kidney. Further mechanism investigation is warranted, as curcumin may be a potentially effective treatment for S-AKI in humans.

\section{Acknowledgments}

This study was sponsored by the National Natural Science Foundation of China (NSFC) (Nos. 81971635 and 82001817).

\section{Disclosure}

The authors declare that there is no conflict of interest associated with this study.

\section{References}

1. Kirsi-Maija, Kaukonen, Michael, et al. Systemic inflammatory response syndrome criteria in defining severe sepsis. $N$ Engl $J$ Med. 2015;372(17):1629-1638.

2. Singbartl K, Kellum JA. AKI in the ICU: definition, epidemiology, risk stratification, and outcomes. Kidney Int. 2012;81(9):819-825. doi:10.1038/ki.2011.339

3. Peerapornratana S, Manrique-Caballero CL, Gómez H, et al. Acute kidney injury from sepsis: current concepts, epidemiology, pathophysiology, prevention and treatment. Kidney Int. 2019;96(5):1083-1099.
4. Bouchard J, Acharya A, Cerda J, et al. A prospective international multicenter study of AKI in the Intensive Care Unit. Clin J Am Soc Nephrol. 2015;10(8):1324-1331. doi:10.2215/CJN.04360514

5. Hoste EA, Bagshaw SM, Bellomo R, et al. Epidemiology of acute kidney injury in critically ill patients: the multinational AKI-EPI study. Intensive Care Med. 2015;41(8):1411-1423. doi:10.1007/ s00134-015-3934-7

6. Rivers EP, Coba V, Whitmill M. Early goal-directed therapy in severe sepsis and septic shock: a contemporary review of the literature. Curr Opin Anaesthesiol. 2008;21(2):128-140. doi:10.1097/ACO.0b013e3282f $4 \mathrm{db} 7 \mathrm{a}$

7. Kellum JA, Chawla LS, Keener C, et al. The effects of alternative resuscitation strategies on acute kidney injury in patients with septic shock. Am J Respir Crit Care Med. 2016;193(3):281-287. doi:10.1164/rccm.201505-0995OC

8. Holthoff JH, Wang Z, Seely KA, et al. Resveratrol improves renal microcirculation, protects the tubular epithelium, and prolongs survival in a mouse model of sepsis-induced acute kidney injury. Kidney Int. 2012;81(4):370-378. doi:10.1038/ki.2011.347

9. Murugan R, Wen X, Shah N, et al. Plasma inflammatory and apoptosis markers are associated with dialysis dependence and death among critically ill patients receiving renal replacement therapy. Nephrol Dialysis Transplant. 2014;29(10):1854-1864. doi:10.1093/ ndt/gfu051

10. Lundy DJ, Trzeciak S. Microcirculatory dysfunction in sepsis. Crit Care Nurs Clin North Am. 2011;25(4):721-731.

11. Benes J, Chvojka J, Sykora R, et al. Searching for mechanisms that matter in early septic acute kidney injury: an experimental study. Crit Care. 2011;15(5):R256. doi:10.1186/cc10517

12. Wang ZH, Deng LH, Chi CW, et al. A preclinical systematic review of curcumin for protecting the kidney with ischemia reperfusion injury. Oxid Med Cell Longev. 2020;2020(11):1-17. doi:10.1155/ 2020/8857906

13. Goel A, Aggarwal BB. Curcumin, the golden spice from Indian saffron is a chemosensitizer and radiosensitizer for tumors and chemoprotector and radioprotector for normal organs. Nutr Cancer. 2010;62(7):919-930.

14. Kar F, Hacioglu C, Senturk H, et al. Curcumin and LOXblock-1 ameliorate ischemia-reperfusion induced inflammation and acute kidney injury by suppressing the semaphorin-plexin pathway. Life Sci. 2020;256:118016. doi:10.1016/j.lfs.2020.118016

15. Zhang J, Tang L, Li GS, Wang J. The anti-inflammatory effects of curcumin on renal ischemia-reperfusion injury in rats. Ren Fail. 2018;40(1):681-687.

16. Avila-Rojas SH, Tapia E, Briones-Herrera A, et al. Curcumin prevents potassium dichromate (K2Cr2O7)-induced renal hypoxia. Food Chem Toxicol. 2018;121:472-482. doi:10.1016/j.fct.2018.09.046

17. Wu T, Marakka TB, Ye Y, et al. Curcumin attenuates both acute and chronic immune nephritis. Int $J$ Mol Sci. 2020;21(5):1745. doi:10.3390/ijms21051745

18. Hubbard WJ, Choudhry M, Schwacha MG, et al. Cecal ligation and puncture. Shock. 2005;24(Suppl 1):52-57. doi:10.1097/01. shk.0000191414.94461.7e

19. Murphy KJ, Rubin JM. Power Doppler: it's a good thing. Semin Ultrasound CT MR. 1997;18(1):13-21. doi:10.1016/S0887-2171(97) 90034-2

20. Feigenbaum H. Role of M-mode technique in today's Echocardiography. J Am Soc Echocardiogr. 2010;23(3):240-257. doi:10.1016/j.echo.2010.01.015

21. Ayache B, de Jong N. WFUMB safety symposium on echo-contrast agents: nature and types of ultrasound contrast agents. Ultrasound Med Biol. 2007;33(2):187-196. doi:10.1016/j.ultrasmedbio.2006.07.008

22. Kellum JA, Lameire N, Aspelin P, et al. Kidney Disease: Improving Global Outcomes (KDIGO) Acute Kidney Injury Work Group. KDIGO Clinical Practice Guideline for Acute Kidney Injury. Kidney Int Suppl. 2012;2(1):1-38. 
23. Anderberg SB, Luther T, Frithiof R. Physiological aspects of Toll-like receptor 4 activation in sepsis-induced acute kidney injury. Acta Physiol. 2017;219(3):573-588. doi:10.1111/apha.12798

24. Kellum JA, Prowle JR. Paradigms of acute kidney injury in the intensive care setting. Nat Rev Nephrol. 2018;14(4):217-230. doi:10.1038/nrneph.2017.184

25. Bellomo R, Kellum JA, Ronco C. Acute kidney injury. Lancet. 2012;380(9843):756-766. doi:10.1016/S0140-6736(11)61454-2

26. Verma SK, Molitoris BA. Renal endothelial injury and microvascular dysfunction in acute kidney injury. Semin Nephrol. 2015;35 (1):96-107.

27. Gomez H, Ince C, Backer DD, et al. A unified theory of sepsis-induced acute kidney injury: inflammation, microcirculatory dysfunction, bioenergetics, and the tubular cell adaptation to injury. Shock. 2014;41(1):3-11. doi:10.1097/SHK.0000000000000052

28. Post EH, Kellum JA, Bellomo R, et al. Renal perfusion in sepsis: from macro- to microcirculation. Kidney Int. 2017;91(1):45-60. doi:10.1016/j.kint.2016.07.032

29. Luo CJ, Zhang FJ, Zhang L, et al. Mesenchymal stem cells ameliorate sepsis-associated acute kidney injury in mice. Shock. 2014;41 (2):123-129. doi:10.1097/SHK.0000000000000080

30. Maiden MJ, Otto S, Brealey JK, et al. Structure and function of the kidney in septic shock: a prospective controlled experimental study. Am J Respir Crit Care Med. 2016;194(6):692-700. doi:10.1164/ rccm.201511-2285OC
31. Lankadeva YR, Kosaka J, Iguchi N, et al. Effects of fluid bolus therapy on renal perfusion, oxygenation, and function in early experimental septic kidney injury. Crit Care Med. 2018;47(1):e36-e43. doi:10.1097/CCM.0000000000003507

32. Kosaka J, Lankadeva YR, May CN, et al. Histopathology of septic acute kidney injury: a systematic review of experimental data. Crit Care Med. 2016;44(9):e897-903. doi:10.1097/CCM.0000000000001735

33. Wang L, Mohan C. Contrast-enhanced ultrasound: a promising method for renal microvascular perfusion evaluation. J Transl Int Med. 2016;4(3):104-108. doi:10.1515/jtim-2016-0033

34. Anatole H, Nicolas G, Samy F, et al. Acute kidney injury is associated with a decrease in cortical renal perfusion during septic shock. Crit Care. 2018;22(1):161. doi:10.1186/s13054-018-2067-0

35. Backer DD, Donadello K, Taccone FS, et al. Microcirculatory alterations: potential mechanisms and implications for therapy. Ann Intensive Care. 2011;1(1):1-8. doi:10.1186/2110-5820-1-27

36. Xia J, Wang H, Zhang QM, et al. The therapeutic effect of curcumin in male albino rats and its putative mechanisms on cerebral microvascular flow. Brain Res. 2016;1642:131-135. doi:10.1016/j. brainres.2016.03.022

37. Zhu JN, Zhang Y, Li XM, et al. Doppler-based renal resistive index for prediction of acute kidney injury in critically ill patients: a systematic review and meta-analysis. Adv Ultrasound Diagn Ther. 2021;03:183-196.

\section{Publish your work in this journal}

Drug Design, Development and Therapy is an international, peerreviewed open-access journal that spans the spectrum of drug design and development through to clinical applications. Clinical outcomes, patient safety, and programs for the development and effective, safe, and sustained use of medicines are a feature of the journal, which has also been accepted for indexing on PubMed Central. The manuscript management system is completely online and includes a very quick and fair peer-review system, which is all easy to use. Visit http://www. dovepress.com/testimonials.php to read real quotes from published authors. 